\title{
Realization of ITS Applications through Mapping Technologies: A Survey of Advanced Traveler Information Systems
}

\author{
Patrick A. Campbell, Joseph P. Havlicek, Alan R. Stevenson, and Ronald D. Barnes
}

\begin{abstract}
ITS applications throughout the United States use a variety of mapping technologies to efficiently relay traffic information to both traffic agents and the general public. This paper describes the findings of a nationwide survey of the mapping approaches taken by traveler information websites. The technologies used within the Oklahoma ITS architecture are also presented within the context of this survey. The mapping technologies discussed include ArcGIS, MapServer, HTMLbased techniques, Google Maps, Bing Maps, and OpenLayers. The tradeoffs that would be considered when evaluating the use of mapping technologies in new or existing ITS applications are presented for comparison.
\end{abstract}

\section{INTRODUCTION}

Mapping technologies are a critical element enabling ITS applications to quickly and efficiently disseminate information to travelers so that they can make intelligent decisions based on the status of roadways. This group of users includes both those who manage roadways as well as those who navigate them. The former primarily use ITS applications that assist in Traffic Management Center (TMC) operations; the latter absorb traffic information that is made publicly available via Advanced Traveler Information Systems (ATIS). ITS applications that are used in TMC's are typically specialty, proprietary software whereas an ATIS more commonly consists of both standard web pages and mobile device applications. The overlay of traffic data on interactive maps is often used within each of these applications as an effective method of communicating information from roadside sensors and other sources of information. However the underlying technologies that drive such maps include a range of different software tools, data sources, and services [1].

In this paper, we examine mapping technologies that have found wide usage in ITS in the United States, with a focus on mapping technologies used in web-based ATIS applications. The mapping technologies considered in this paper reflect a survey of mapping technologies examined in the development of the Oklahoma ITS architecture as well as those used in public traffic and roadway systems throughout the nation. Oklahoma's ITS architecture includes a novel virtualized Traffic Management Center [2] as well as a public ATIS website [3], and several of the state's ITS components

This work was supported in part by grants from the Oklahoma Department of Transportation and the Oklahoma Highway Safety Office.

P. Campbell, J. Havlicek, and R. Barnes are with the University of Oklahoma ITS Laboratory, School of Electrical and Computer Engineering, Norman, OK 73019, USA. \{pacampbel1, joebob, ron\}@ou . edu

A. Stevenson is with the the Oklahoma Department of Transportation, Technology Services Division, Oklahoma City, OK 73105, USA. astevensoneodot.org have required the analysis, selection, and implementation of effective mapping approaches. The specific focus of the survey was to examine the Advanced Traveler Information Systems for cities and states across the United States with an aim to determine the most common mapping technologies used and their distinctive characteristics comprising their advantages and disadvantages.

The mapping technologies described in this work include the mapping tools, services, and techniques of ArcGIS, MapServer, HTML-based mapping techniques, Google Maps, Bing Maps, and OpenLayers. While not necessarily comprehensive, this list represents a best practices approach to the selection and implementation of mapping options for ITS applications. The list is necessarily biased towards web-based GIS solutions which require open connectivity to the world wide web, and in which components of the mapping solutions can be located on remote machines. Some environments that have more restricted internet access or need more powerful map processing capabilities, such as a TMC, may benefit more from localized GIS solutions that can run independently on a single machine. However, in these environments several of the same technologies covered here are still readily applied, though the preferred solution may differ from that selected for a web-based environment.

The following sections present each mapping technology considered, and describe advantages, disadvantages, and use cases for the technology. The results of the nationwide survey are then presented, and our own selections and conclusions are described in a presentation of Oklahoma's approach to the inclusion of mapping technology within its ITS components.

\section{ARCGIS}

A common commercial mapping technology available for ITS applications exists in the range of products produced by the Environmental Systems Research Institute (ESRI). These mapping products are referred to in aggregate as ArcGIS, and are divided into products specialized for desktop, mobile, server, and online use.

\section{A. Advantages}

Founded in 1969 [4], ESRI is a well-established and trusted company that has come to be widely used and supported within the geographic information and analysis community. The range of products offered by the company as well as the company's history can make ArcGIS an attractive mapping solution depending on an organization's existing licenses, personnel, and data. The ArcGIS-family 
product specifically intended to be used in the implementation of online mapping services is ArcGIS Online. Within ArcGIS Online there are different implementations, including Javascript, Flex (a Flash-based language), and Silverlight (a Microsoft alternative to Flash), each making use of a different programming language to implement mapping features.

Each of these options can be easily integrated into standard web programming practices, making ArcGIS Online an attractive option in terms of development and maintenance costs. In addition, the Flex option supports a unique timeline feature not found in other mapping technologies. Using this feature, map elements can be interactively displayed in chronological order.

ArcGIS Online could potentially be a cost-effective solution for many organizations as the product can be used for free for limited, non-commercial external use or in conjunction with an existing ArcGIS Server license [5]. Once licensed, the product can also be used with existing GIS data supplied by ESRI. ArcGIS Online can furthermore be used to display an organization's own GIS data along with data imported from other services including Google or OpenStreetMap [6]. Technical support is also provided with a license, which can be a valuable resource.

Because of broad adoption of ArcGIS for many GIS applications, many organizations may already have personnel who are certified for ArcGIS software. This can increase the costeffectiveness to the organization in terms of development and maintenance, as such personnel will be able to readily make use of ArcGIS Online.

ESRI also offers attractive options for applications which may need to be run within private or restricted networks. Products such as ArcGIS Server and ArcGIS Engine may be used to incorporate GIS functionality into stand-alone applications such as those used in a TMC.

\section{B. Considerations}

Although ArcGIS is potentially cost effective, license fees can still be substantial. As of March 2012, private or commercial deployment license fees can cost $\$ 2000$ to $\$ 4000$ per year for ArcGIS Web Mapping API access [7]. For stand alone applications, ArcGIS Engine may be licensed throughout an organization for a yearly subscription fee on the order of $\$ 2000$ per year [8].

In the case where an organization does not have personnel who are certified or at least familiar with ESRI products, the use of ArcGIS, especially as a component of a custom application, can require significant training on ESRI products and methodology.

\section{Example uses in ITS applications}

ArcGIS has been used in organizations worldwide in a large variety of projects requiring geospatial analysis. Within ITS, these include 3-D mapping within a TMC [10], emergency evacuation planning [11], and multimodal traveler information websites worldwide [12]. Each of the three ArcGIS Online options described above were identified in

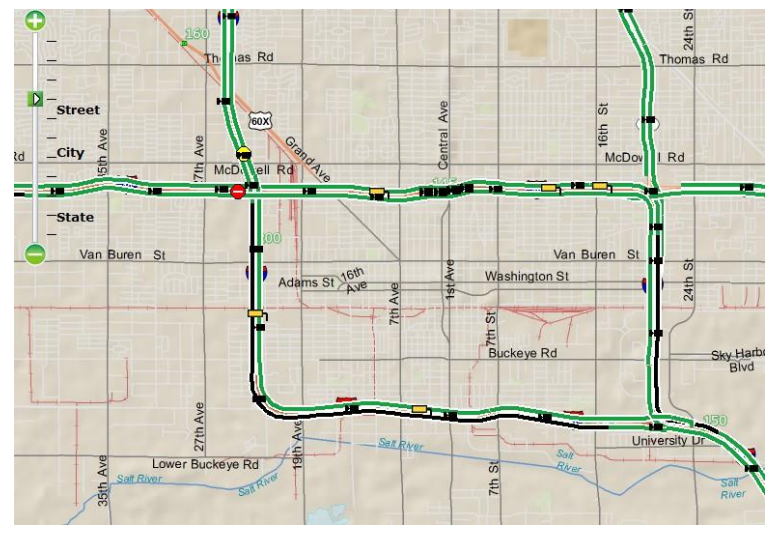

Fig. 1. Map generated using ArcGIS in Arizona's 511 site [9]. Displayed are cameras, traffic speeds, road closures, and message signs in downtown Pheonix.

our survey of Advanced Traveler Information Systems, including use of the Javascript option in Arizona [9], North Dakota [13] and Austin [14], the Flex option in Missouri [15] and New Mexico [16], and the Silverlight option in Illinois [17]. An example of a map generated in Arizona's 511 website is shown in Fig. 1.

\section{MAPSERVER}

MapServer [18] is an open source mapping software developed by the University of Minnesota that generates map images from GIS data and has found use in a few ITS applications.

\section{A. Advantages}

Because it is a free-to-use open source mapping technology, MapServer is a very cost-effective solution for mapping applications. Also due to its open source nature, developers have complete control over the implementation and use of their maps; this control extends as far as the ability to modify the source code of the MapServer libraries. This provides freedom to develop individual solutions to unique or unforeseeable problems.

MapServer libraries provide considerable functionality. They contain capabilities to incorporate existing GIS data, giving organizations the ability to make use of data they may already own. Extensive documentation and examples are also provided online to help developers use the libraries. These libraries are actively maintained and developed, providing additional functionality over time.

A component of the MapServer project is the MapScript PHP libraries that provide MapServer functionality to PHP code often used within websites. This provides a solution that integrates well with standard web programming practices as well as server-side components such as databases.

\section{B. Considerations}

Even with documentation and examples, MapServer software has a significant learning curve that can increase the costs of development and maintenance. The use of MapServer technology can be more complex than other 


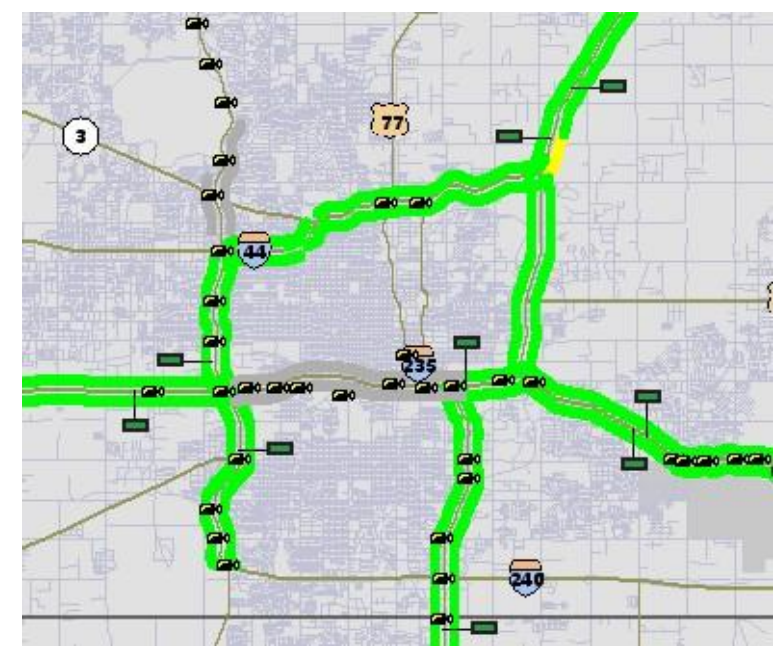

Fig. 2. Map generated using MapServer in Oklahoma ATIS [3]. Displayed are traffic speeds, cameras, and message signs in the Oklahoma City area.

mapping technologies, potentially resulting in considerably longer implementation times than encountered when using commercial mapping systems. The complexity also leads to maintenance difficulties as modifications to the underlying complex code may often require the implementor to relearn code even to make minor modifications.

MapServer is, strictly speaking, a set of mapping libraries. An organization using MapServer in ITS applications must already have or otherwise procure the needed GIS data for any desired maps. This GIS data must be obtained for any map elements which need to be drawn, including roadways and county lines. More advanced mapping system features must be developed by users of the libraries. For example, the Oklahoma ATIS includes a caching system to reduce server lag [3]. Additionally, interactive mapping features such as mouse-driven pan and zoom operations may need to be implemented by application developers. This requirement to implement functionality found in other mapping technologies can further reduce the cost effectiveness of MapServer-based ITS applications.

\section{Example uses in applications}

MapServer can be an extremely attractive solution when recurring license fees for commercial mapping solutions are prohibitively expensive. As examples, the solution has been employed within the Oklahoma ITS architecture [2], [3], within ship monitoring systems [19], archaeological digital libraries [20], and in an urban disaster information system [21]. Mapserver is also used to disseminate roadway weather information in Alaska [22]. Fig. 2 shows a map generated by MapServer in Oklahoma's ATIS.

\section{HTML SOLUTIONS}

Several online ITS applications rely on elements of standard HTML to implement maps which are capable of displaying traffic and roadway information. While not nearly as feature-rich as most mapping systems, HTML solutions can offer a simple and economic solution to the problem of distributing information to online users. Two dominant HTML techniques were found to be used within traveler information sites, each primarily making use of specific HTML elements.

The first technique makes use of the map and area elements, which can be used to create a basic user interactive map. The map element contains the entire map, then each area element defines a region within the map element, as well as the action to be taken when the user clicks on that region. In practice, sites display a map in the background, then define area elements for each roadway device on the map.

The second technique makes use of the image element to overlay device images on a map image. An image element is used to display the map background, then additional image elements are added to the page, with their page positions precisely defined so that they appear in the correct place over the map. These images can then be clicked to interact with the map.

An additional unique solution was found in Honolulu's traffic site [23] which primarily used HTML table elements. In this solution, an HTML table was defined for the entire map region and was composed of a large number of small cells in a grid. Each table cell then contained a small image of the map at that grid location. Users could then click on individual cells of the table in order to interact with the map.

\section{A. Advantages}

The primary advantage of HTML solutions is that they are completely free to use. HTML is a ubiquitous standard used throughout the world wide web that certainly requires no license fees. The HTML solutions can also be relatively simple. With the use of these solutions, no mapping system expertise is required and a larger pool of developers can develop and maintain the site, even those with limited technical or programming knowledge.

HTML based solutions are also compatible across a wide range of users. Because the HTML standard has long been in use, websites using these solutions will not have the kind of compatibility problems, such as users who do not have JavaScript turned on or do not have Flash installed, that may hinder other mapping implementations.

\section{B. Considerations}

HTML based solutions are generally feature-poor compared to the newest mapping solutions available, lacking useful features that users have come to expect when viewing and analyzing geospatial data. Additionally, each of the HTML techniques above suffer potential limitations when traffic information overlaps on the screen.

Although the solutions are straightforward in that the individual elements used are basic HTML tags, in practice complete applications can be quite complex. In order to make a functioning map that distributes the desired information, these tags must be utilized in creative, and ultimately complex ways. Any site attempting to provide dozens or hundreds of pieces of real-time traffic information 


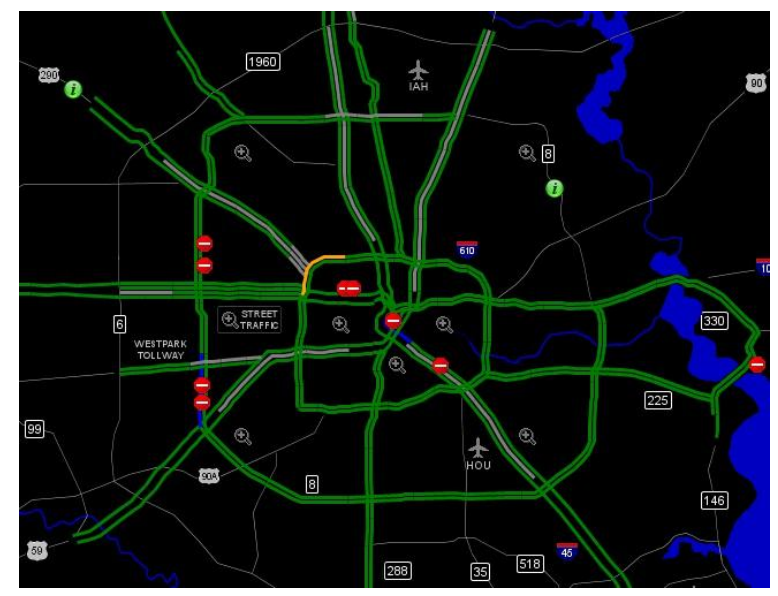

Fig. 3. Map generated using HTML in Houston Transtar [26]. Displayed are traffic speeds and road closures in the Houston area.

will contain copious amounts of HTML code that must be automatically generated by server-side programs. As in other mapping technologies, such an implementation will require experienced programmers. In several sites, map elements may have been manually placed on map images without the use of server-side programs. However, these pages can be very tedious to set up and difficult to maintain.

Lastly, HTML-based maps often do not look as attractive as current mapping technologies. The polish and ease of use that users have come to expect from technologies such as Google Maps is generally not found in HTML-based maps, making it difficult for websites which use them to achieve a modern, professional look and feel.

\section{Example uses in ITS applications}

The technology used in HTML based solutions predates technologies such as Google maps. In the past, this option was likely considerably more attractive as an inexpensive way to distribute information. In many cases, the sites using HTML-only techniques are likely sites that were developed several years ago and have not been revised to use newer mapping technology. In many cases, as they still fulfill their purpose reasonably well, and it is likely not justifiable to update them. Examples of traveler information sites using HTML-based maps include Arkansas [24] and Los Angeles [25].

In some instances, site designers feel that newer mapping technologies are too feature-rich and are unnecessarily confusing to end users. The basic HTML solution offers a simple solution that quickly communicates only needed information. An interesting example of this lies within the traveler information website for Houston [26], which offers both HTML-based and Bing mapping solutions. The HTML solution has a clear black background with highways and devices shown in clear and distinct colors, as seen in Fig. 3. This map at first appears uninviting, but upon comparison with the corresponding Bing map, it is noted that the wealth of additional information in the Bing map, including cities, streets, businesses, and geographic features, can overwhelm the traffic information. Especially if a user is familiar with the relevant roadways, the basic HTML-based map may present information more efficiently.

\section{GoOgle Maps AND BING MAPS}

Prior to the release of Google Maps by Google Inc. in 2005 [27], ITS applications employed a wide range of mapping technologies including those discussed in previous sections. However, with the release of Google Maps and the subsequent release of Microsoft Bing Maps, a large number of ITS applications, in particular ATIS web sites, have moved towards the use of these highly interactive, internet-based mapping systems. Although there are several differences between the two systems, both offer an intuitive user interface that allows users to quickly navigate geographic information, and both use standard web development practices making them easy to integrate into websites.

\section{A. Advantages}

The popularity of Google maps and Bing maps among developers and the general public has been a result of several attractive features. The mapping systems are built upon and used by writing JavaScript. JavaScript is commonly used and easily supported within websites. The Application Programming Interface (API) for each system provides a high level of implementation control while remaining simple and compact. As a result developers are able to easily implement desired functionality into websites that require comparatively little maintenance.

Furthermore, with the substantial initial support of two large corporations for the systems, the excellent, easy to use map interface quickly became one that is recognized and widely used by most internet users. The map interface offered a novel and immediately attractive functionality to the general population, and enough were impressed upon first usage to ensure its mainstream acceptance.

Google maps and Bing maps have also been implemented in many websites because they are free to use for public, non-commercial, or development sites.

\section{B. Considerations}

For many websites, each of these mapping systems can incur a sizable fee for deployment. Although both are free for public, non-commercial sites, there are relatively low usage limits placed on free deployments. In addition, licenses may be required for government agencies.

Both systems have several pricing options, but the estimated cost of a license for each is on the rough order of $\$ 10000$ per year [28]-[30]. A license from Google entitles an organization to a set number of page views, with plans available for varying numbers of page views. In the case of Bing Maps, licenses are based on numbers of transactions, where one transaction is considered to be 8 map tile loads, 1 geocoding operation, or 1 route-finding operation. Plans are also available that are based on a fixed number of users or number of tracked assets. 


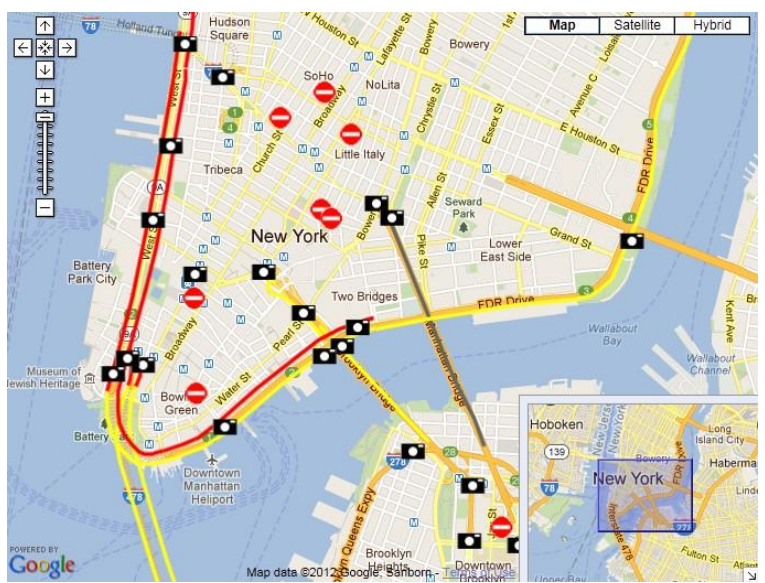

Fig. 4. Map generated using Google Maps in New York's 511 site [31]. Displayed are traffic speeds, cameras, and road closures in lower Manhattan.

\section{Example uses in ITS applications}

In many cases, state and city traffic agencies have either been able to qualify for free usage of Google or Bing maps or have decided that the benefits of the mapping systems are worth the deployment fees. Google mapping systems have also been used in ITS applications such as a traffic violation alert system [32], a transit information system [33], and an arterial information system [34]. Some traveler information sites that make use of Google maps are New York [31], California [35], Kansas City [36], Colorado [37], and Florida [38]. Sites that use Bing maps include DallasForth Worth [39], Kansas [40], Seattle [41], and Ohio [42]. Fig. 4 shows a map generated by Google in New York's 511 website.

\section{OPENLAYERS}

Released in 2006, OpenLayers [43] is an open source JavaScript based mapping technology that offers many of the same map interface features as Google and Bing maps. A potential source of map data that may be used with OpenLayers is OpenStreetMap [6], a relatively new initiative drawing on mass-user contribution concepts similar to those used in Wikipedia.

\section{A. Advantages}

The primary advantage of OpenLayers, as with other open source projects, is that it is completely free to use. The project also integrates well with standard web programming practices since it is based on JavaScript, and the same 'clean' code benefits of Google and Bing maps can be realized using OpenLayers. OpenLayers is also continually developed, and can be used to display any existing GIS data that an organization may already have.

OpenStreetMap is a free-to-use map data project that may be used in conjunction with OpenLayers as a source of map data. The maps in OpenStreetMap can be edited at will by anyone, meaning that the maps can in theory improve over time, and that an organization may fix map inaccuracies on the fly.

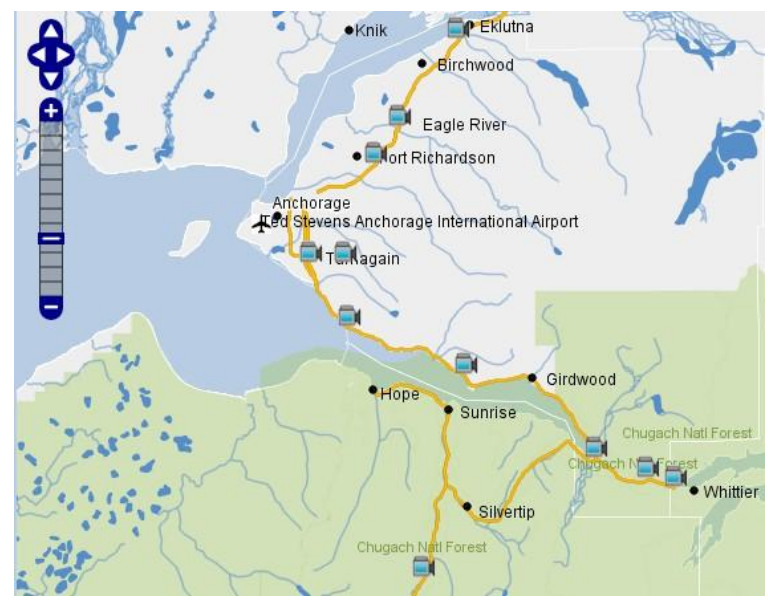

Fig. 5. Map generated using OpenLayers in Alaska's 511 site [44]. Displayed are cameras in the Anchorage region.

\section{B. Considerations}

OpenLayers is map interface technology only; the underlying map data must still be provided by the implementor. This can be an economic advantage when an organization already owns relevant geospatial data, but often the data owned by or available to organizations lacks the depth of that collected by corporations such as Google, Microsoft, or ESRI. Even if high quality data is available, it can still be difficult to create polished maps that compare to the clarity and aesthetic favorability that most web site visitors have grown accustomed to in Google and Bing maps.

OpenStreetMap offers a comprehensive and visually pleasing data set, however the primary consideration for an organization that may wish to rely on OpenStreetMap is that the map data can be edited by anyone, meaning that the organization has little control over the quality of the maps. Although users will gradually improve map quality, many users may accidentally or even purposefully introduce errors and ambiguities into the map data. This lack of quality control may prove to be unacceptable on public ATIS sites upon which the public relies for accurate information. In more private applications, such as those used internally within a TMC, the questionable quality of the maps may be acceptable, especially given the low cost of the solution. However, in applications that operators rely on to manage traffic, or especially to direct emergency response, questionable map quality is still likely unacceptable.

\section{Example uses in applications}

Although OpenStreetMap was not found in any public traveler information sites, OpenLayers proved to be an attractive solution for traveler information sites in Alaska [44], Nevada [45], and South Dakota [46]. OpenLayers has also found use in diverse projects such as a water quality management system [47] and a ship monitoring system [19], and has been discussed in the context of GIS processing and optimization in [48] and [49]. Fig. 5 shows a map generated by OpenLayers in Alaska's 511 website. 
TABLE I

MAPPING TEChNOLOGy Distribution FOR NATIONWIDE TraVEleR INFORMATION SITES. STATE SITES REFLECT THE PREDOMINANT SITES FOUND FOR EACH STATE. ONE SITE [26] OFFERED TWO MAPPING SOLUTIONS.

\begin{tabular}{|c|c|c|c|}
\hline \multicolumn{2}{|c|}{ State Sites Only } & \multicolumn{2}{c|}{ All Sites } \\
\hline Google Maps & 29 & Google Maps & 38 \\
Bing Maps & 5 & Bing Maps & 8 \\
OpenLayers & 3 & HTML (map tag) & 7 \\
ArcGIS (Javascript) & 2 & HTML (image tag) & 5 \\
ArcGIS (Flex) & 2 & ArcGIS (Javascript) & 4 \\
HTML (map tag) & 2 & ArcGIS (Flex) & 3 \\
HTML (image tag) & 2 & OpenLayers & 3 \\
ArcGIS (Silverlight) & 1 & Mapserver & 3 \\
Mapserver & 1 & ArcGIS (Silverlight) & 2 \\
HTML (table tag) & 1 & Unknown & 2 \\
Oracle Maps & 1 & HTML (table tag) & 1 \\
Telogis & 1 & Oracle Maps & 1 \\
& & Telogis & 1 \\
& & TLCGIS & 1 \\
& & ArcIMS & 1 \\
\hline
\end{tabular}

\section{CONCLUSIONS}

In summary, we note the observed distribution of the mapping technologies found in our survey. We also describe Oklahoma's current usage of these technologies and provide some insight into the evaluation of the technologies for ITS applications in the State of Oklahoma.

\section{A. Survey Results}

Our survey was carried out on traffic and roadway information websites collected into the National Traffic and Road Closure Information online resource maintained by the Federal Highway Administration [50]. In total, 79 sites were surveyed and analyzed to determine the technologies in use as of March, 2012. Table I summarizes the resulting distribution of mapping technologies found during the survey.

From Table I, Google Maps has become the dominant mapping technology for traveler information websites in the United States. Although many ITS applications have historically used a variety of mapping technologies, the ease of use, support for, and cost-effectiveness of Google Maps has made it the "go-to" solution for many sites. Furthermore, many sites which had previously relied on alternate technology may have decided, for the above reasons, to revise their site to accommodate Google maps. The second most common mapping approach in the distribution is Bing Maps, which offers many of the same advantages as Google Maps.

The popularity of Google maps is also somewhat explained by consultant work utilized by several states to implement their traveler information sites. These consultants often choose to work with Google. In particular, Castle Rock Consulting [51] has developed a standard 511 traffic information interface, which uses Google maps, and which was used in 9 of the sites surveyed, including those for Idaho [52], Louisiana [53], Maine [54], and Iowa [55]. This approach may prove to be an attractive option for other states as well, as the websites are clear and informative and the website implementation and maintenance is the responsibility of the third party firm. However, this standard interface acts as a front-end for the Condition Acquisition and Reporting System (CARS), which is a roadway reporting initiative by Castle Rock. As such, any organization utilizing this option may necessarily need to deeply invest in the infrastructure of the CARS system. While this may not be optimal for states with an existing ITS infrastructure, contracting the management of ITS infrastructure can be an excellent ITS strategy for many states.

A separate, readily identifiable consulting firm was Meridian Environmental Technology Inc, which is in the business of, among other things, designing custom traveler information sites for a variety of agencies. Meridian offers flexible solutions, tailoring the sites to state's infrastructure and demands. The resulting sites are not nearly as consistent, and instead show considerable variety. Meridian is responsible for 7 of the sites surveyed, and made use of several different mapping technologies including Bing (Kansas) [40] OpenLayers (Nevada) [45] ArcGIS (North Dakota) [13], and Google (Wyoming) [56].

It should be noted that there also exist a small number of additional nationwide traffic information sites that use their own mapping solutions. These sites endeavor to provide traffic information for cities throughout the nation, and include Traffic.com [57], Yahoo Traffic [58], and Beat The Traffic [59].

Although these results reflect only mapping technologies found for nationwide ATIS sites, the same mapping technologies can be applied to applications throughout an ITS infrastructure, including those found within TMCs. The distribution of the most useful technologies may be different depending on the economic and network constraints of the applications. In particular, private network constraints within TMCs may prevent the use of web-based services such as Google and Bing maps.

\section{B. Mapping approaches in Oklahoma ITS Applications}

Oklahoma's approach to a statewide ITS has included the implementation of mapping technologies in both TMC and ATIS components. Over ten years ago, Oklahoma adopted a novel ITS architecture consisting of a virtualized statewide TMC built on a private fiber optic network linking highway devices to controlling stations located at stakeholder agencies throughout the state [2]. Pathfinder, an ATIS component, was added to this architecture, acting as a gateway through which select information from the private network could be delivered to the public internet [3]. The Oklahoma ATIS was also developed to include an access-restricted portion of the site that can be accessed by state traffic engineers and that displays additional device information and provides additional control capabilities.

Both the Oklahoma ATIS and virtualized TMC applications were developed using MapServer technology, specifically the MapScript PHP libraries. This technology provided the state with a free mapping solution that could make use of the state's existing GIS libraries, including roadways, county lines, bodies of water, and city limits. Additionally, the 
technology provided a mapping method that could be used in both the public and internal sections of the ITS architecture without incurring license fees and that integrated well with the other open source technologies used within Oklahoma ITS such as Linux, Apache, and MySQL. In particular, at the time that the Oklahoma ITS architecture was first developed, the cost and compatibility benefits of MapServer technology were enough to justify its use in the implementation of the state's ITS applications.

However, recently a proposal has been made within the state to rewrite applications to make use of Google or Bing maps. Because of the complexity of the state's MapServer solution, the maintenance of its ITS applications has increased in difficulty and has become more time-intensive. In particular, this difficulty has been seen as new features have been added. It has been challenging to implement and keep up with interactive mapping features such as dynamic retrieval of map data and mouse-driven map pan and zoom. Finally, the visual style of the maps included in these applications has started to appear outdated compared to the commercial maps that users have become accustomed to seeing on the web. The state-owned GIS data does not provide some of the information that is included in Google and Bing maps such as satellite imagery, street names, business locations, and street level view.

As a result, a pilot project was implemented in which the Oklahoma ATIS application was rewritten to utilize Bing Maps. This resulted in several system improvements including 'cleaner' system code, increased maintainability, and greatly enhanced user interactivity with traffic information. The main impediment to deployment of the new ATIS was the licensing required for deployment. Although the ATIS is non-commercial and public, Oklahoma's ATIS has a password-protected version. Because of other applications demanding mapping technologies, the state has obtained a license for Google Maps, and a new Google Maps version of the Oklahoma ATIS has been implemented and will be deployed soon. Fortunately the similarities between Google and Bing maps have facilitated development and retained the previous system improvements.

The controlling stations within Oklahoma's virtualized TMC still use the original MapServer technology. The use of internet-based mapping services presents security concerns within the private network. In addition, relying on third-party, internet-provided resources for core TMC functionality may not be desirable, especially in emergency situations. However, with future development of the Oklahoma Virtual TMC, we intend to explore enhancement of this control software through the use of improved mapping technology such as OpenLayers, ArcGIS, or updated MapServer libraries.

\section{REFERENCES}

[1] I. Skog and P. Handel, "In-car positioning and navigation technologies - A survey," IEEE Transactions on Intelligent Transportation Systems, vol. 10, no. 1, pp. $4-21$, March 2009.

[2] B. Kilani, E. Vorakitolan, J. Havlicek, M. Tull, and A. Stevenson, "Distributed ITS control and the Oklahoma virtual TMC," in Proceedings of the 12th International IEEE Conference on Intelligent Transportation Systems, Oct. 2009, pp. 785-790.
[3] P. Campbell, J. Junger, J. Havlicek, A. Stevenson, and R. Barnes, "Pathfinder: Oklahoma's advanced traveler information system," in Proceedings of the 14th International IEEE Conference on Intelligent Transportation Systems, Oct. 2011, pp. 402-407.

[4] ESRI. About Esri - company profile. Visited March, 2012. [Online]. Available: http://www.esri.com/about-esri/index.html

[5] —. ArcGIS Web Mapping APIs. Visited March, 2012. [Online]. Available: http://www.esri.com/software/arcgis/web-mapping/pricing. html

[6] OpenStreetMap. The free wiki world map. Visited March, 2012. [Online]. Available: http://www.openstreetmap.org/

[7] ESRI. How can I get access to the ArcGIS Web APIs. Visited March, 2012. [Online]. Available: http://events. esri.com/uc/QandA/index.cfm?fuseaction=answer\&conferenceId= 2a8e2713-1422-2418-7f20bb7c186b5b83\&questionId=2566

[8] — ArcGIS engine overview. Visited March, 2012. [Online]. Available: http://www.esri.com/software/arcgis/arcgisengine/index.html

[9] ADOT. ADOT traffic. Visited March, 2012. [Online]. Available: http://www.az511.com/adot/files/traffic/

[10] S. Yi, Z. Ping, and Y. Yijin, "Smooth navigation of road map in 3d scene," in Proceedings of the 2003 IEEE Intelligent Transportation Systems Conference, vol. 2, Oct. 2003, pp. 1235-1239 vol.2.

[11] Q. Li, X. Yang, and H. Wei, "Integrating traffic simulation models with evacuation planning system in a gis environment," in Proceedings of the 2006 IEEE Intelligent Transportation Systems Conference, Sept. 2006, pp. 590-595.

[12] P. Kumar, V. Singh, and D. Reddy, "Advanced traveler information system for Hyderabad City," IEEE Transactions on Intelligent Transportation Systems, vol. 6, no. 1, pp. 26-37, March 2005.

[13] NDDOT. North Dakota travel information. Visited March, 2012. [Online]. Available: http://www.dot.nd.gov/travel-info

[14] City of Austin. Trafficviewer. Visited March, 2012. [Online]. Available: http://austintexas.gov/GIS/TrafficViewer/

[15] MoDOT. MoDOT traveler information. Visited March, 2012. [Online]. Available: http://maps.modot.mo.gov/timi/

[16] NMDOT. NMRoads. Visited March, 2012. [Online]. Available: http://www.nmroads.com

[17] IDOT. Getting around Illinois. Visited March, 2012. [Online] Available: http://www.gettingaroundillinois.com/

[18] MapTools.org. PHP MapScript. Visited March, 2011. [Online]. Available: http://www.maptools.org/php_mapscript/

[19] X. Z. Nan and L. H. Tao, "Design of ship monitoring system based on WebGIS," in Proceedings of the 2010 3rd International Conference on Information Sciences and Interaction Sciences, June 2010, pp. 6-8.

[20] R. Shen, W. Fan, D. Gorton, N. Vemuri, and E. Fox, "ETANA-GIS: GIS for archaeological digital libraries," in Proceedings of the 6th ACM/IEEE-CS Joint Conference on Digital Libraries, June 2006, p. 379.

[21] K. Chen, J. Rui, and J. Wen, "Development of Shanghai urban disaster information system based on open source WebGIS," in Proceedings of the 19th International Conference on Geoinformatics, June 2011, pp. $1-4$.

[22] Alaska DOT. Road weather information, transportation \& public facilities, state of Alaska. Visited March, 2012. [Online]. Available: http://www.dot.state.ak.us/iways/roadweather/

[23] City of Honolulu. Honolulu traffic cameras. Visited March, 2012. [Online]. Available: http://www1.honolulu.gov/cameras/traffic.htm

[24] AHTD. Arkansas lane closures. Visited March, 2012. [Online]. Available: http://www.arkansashighways.com/lanes/lanemap3.htm

[25] LADOT. Los Angeles city traffic conditions. Visited March, 2012. [Online]. Available: http://trafficinfo.lacity.org/

[26] City of Houston. Houston TranStar. Visited March, 2012. [Online]. Available: http://www.houstontranstar.org

[27] Google. Mapping your way. Visited March, 2012. [Online]. Available: http://googleblog.blogspot.com/2005/02/mapping-your-way. html $\backslash \# ! / 2005 / 02 /$ mapping-your-way.html

[28] _. Google Maps API for business. Visited March, 2012. [Online]. Available: http://www.google.com/enterprise/earthmaps/ maps-faq.html

[29] Microsoft. Bing Maps API licensing and pricing information. Visited March, 2012. [Online]. Available: http://www.microsoft.com/maps/ product/licensing_for_enterprise.aspx

[30] Earthware. Visited March, 2012. [Online]. Available: http://www.earthware.co.uk/blog/index.php/2009/12/ mapping-apis-google-maps-vs-bing-maps-part-2-licensing/ 
[31] NYDOT. 511NY, get connnected to go. Visited March, 2012. [Online]. Available: http://www.511ny.org/

[32] N. Aliane, J. Fernandez, S. Bemposta, and M. Mata, "Traffic violation alert and management," in Proceedings of the 14th International IEEE Conference on Intelligent Transportation Systems, Oct. 2011, pp. 1716-1720.

[33] R. Hoar, "A personalized web based public transit information system with user feedback," in Proceedings of the 13th International IEEE Conference on Intelligent Transportation Systems, Sept. 2010, pp. 1807-1812.

[34] Y.-J. Wu, Y. Wang, and D. Qian, "A Google-map-based arterial traffic information system," in Proceedings of the 2007 IEEE Intelligent Transportation Systems Conference, Oct. 2007, pp. 968-973.

[35] California DOT. Caltrans - Quickmap. Visited March, 2012. [Online]. Available: http://quickmap.dot.ca.gov/

[36] MoDOT, KDOT. Kansas City Scout. Visited March, 2012. [Online]. Available: http://www.kcscout.net

[37] CDOT. Road conditions, speeds, traffic cameras, road closures and road work information. Visited March, 2012. [Online]. Available: http://www.cotrip.org

[38] FDOT. Florida's 511 traveler information system. Visited March, 2012. [Online]. Available: http://www.fl511.com

[39] Texas DOT. Dallas/fort worth traffic conditions. Visited March, 2012. [Online]. Available: http://dfwtraffic.dot.state.tx.us

[40] Kansas DOT. KanRoad 2011-49. Visited March, 2012. [Online]. Available: http://511.ksdot.org/

[41] City of Seattle. Seattle travelers traffic camera maps. Visited March, 2012. [Online]. Available: http://web5.seattle.gov/travelers/

[42] Ohio DOT. Buckeye traffic. Visited March, 2012. [Online]. Available: http://www.buckeyetraffic.org/

[43] OpenLayers. Free maps for the web. Visited March, 2012. [Online]. Available: http://www.openlayers.org/

[44] Alaska DOT. Alaska 511 - transportation \& public facilities. Visited March, 2012. [Online]. Available: http://511.alaska.gov/

[45] Nevada DOT. Safe Travel USA - Nevada. Visited March, 2012. [Online]. Available: http://www.safetravelusa.com/nv/

[46] South Dakota DOT. Safe travel USA - South Dakota. Visited March, 2012. [Online]. Available: http://www.safetravelusa.com/sd/

[47] C. Mullinix, P. Hearn, H. Zhang, and J. Aguinaldo, "Web-based decision support and visualization tools for water quality management in the chesapeake bay watershed," in Proceedings of the 17th International Conference on Geoinformatics, Aug. 2009, pp. 1-6.

[48] E. Wolf and K. Howe, "Web-client based distributed generalization and geoprocessing," in Proceedings of the 2009 International Conference on Advanced Geographic Information Systems Web Services, Feb. 2009, pp. 123-128.

[49] X. Zhang, G. Li, and X. Lan, "Research on WebGIS performance optimization," in Proceedings of the 7th International Conference on Wireless Communications, Networking and Mobile Computing, Sept. 2011, pp. 1-4.

[50] FHWA. National traffic and road closure information. Visited March, 2012. [Online]. Available: http://www.fhwa.dot.gov/trafficinfo/

[51] Castle Rock Consulting. Castle Rock consultants - intelligent transportation specialists. Visited March, 2012. [Online]. Available: http://www.crc-corp.com/

[52] Idaho Transportation Department Traveler Information. Title. Visited March, 2012. [Online]. Available: http://511.idaho.gov

[53] Louisiana DOTD. Louisiana DOT traveler information. Visited March, 2012. [Online]. Available: http://www.511la.org

[54] Maine DOT. Maine DOT 511 highway information. Visited March, 2012. [Online]. Available: http://www.511maine.gov

[55] Iowa DOT. Iowa Department of Transportation Traveler Information. Visited March, 2012. [Online]. Available: http://www.511ia.org

[56] WYDOT. Wyoming travel information map. Visited March, 2012. [Online]. Available: http://map.wyoroad.info/

[57] Traffic.com. Traffic.com. Visited March, 2012. [Online]. Available: http://www.traffic.com/

[58] Yahoo! Yahoo! maps, driving directions, and traffic. Visited March, 2012. [Online]. Available: http://traffic.yahoo.com/

[59] Beat the Traffic. Beat the Traffic traffic maps. Visited March, 2012. [Online]. Available: http://maps.beatthetraffic.com/ 